\title{
iTRAQ-based quantitative proteomic analysis reveals the metabolic pathways of grain chalky occurrence in response to nitrogen topdressing for rice
}

Min Xi ( $\sim$ min8552@126.com )

Anhui Academy of Agricultural Sciences

Wenge Wu

Anhui Academy of Agricultural Sciences

Younzun Xu

Anhui Academy of Agricultural Sciences

Yongjin Zhou

Anhui Academy of Agricultural Sciences

Gang Chen

Anhui Academy of Agricultural Sciences

Yalan Ji

Anhui Academy of Agricultural Sciences

Xueyuan Sun

Anhui Academy of Agricultural Sciences

Research article

Keywords: Rice, iTRAQ, Proteome, Grain chalkiness, Nitrogen fertilizer

Posted Date: August 2nd, 2019

DOI: https://doi.org/10.21203/rs.2.11182/v1

License: (c) (1) This work is licensed under a Creative Commons Attribution 4.0 International License. Read Full License 


\section{Abstract}

Background Grain chalkiness is a highly undesirable quality trait that adversely affects consumer acceptability and lowers the market value. Except for the genotypic variation among different rice cultivars, this chalkiness is readily influenced by application of chemical fertilizers, particularly nitrogen $(\mathrm{N})$ at the late growth stage of rice. However, it is not fully clear on the molecular mechanism underlying the formation of grain chalkiness caused by $\mathrm{N}$ fertilization. Results Using $0 \mathrm{M} 052$ with the chalkiness rate over $90 \%$ as material, experiment was conducted with two $\mathrm{N}$ fertilizer topdressing treatments at the panicle initiation stage, including $108 \mathrm{~kg} \mathrm{~N}$ ha-1 applied $(\mathrm{N}+)$ and without $\mathrm{N}$ topdressing to rice as a control (NO). $\mathrm{N}+$ significantly enhanced the area of endosperm chalkiness and the degree of grain chalkiness. The amylose content of rice grains under $\mathrm{N}+$ was lower than that under N0, while the opposite trend was true for $\mathrm{N}+-$ induced change in grain protein content. Proteomic analysis found that a total of 198 proteins differentially expressed between $\mathrm{N}+$ and N0, including 9 up-regulated proteins and 189 down-regulated proteins for rice plants imposed to $\mathrm{N}+$. Approximately $31.3 \%$ of these differentially expressed proteins (DEPs) involved in N metabolism (protein synthesis, folding, degradation and storage, and amino acid synthesis and catabolism), $21.7 \%$ of DEPs belonged to carbohydrate metabolism (glycolysis, tricarboxylic acid cycle, pentose phosphate pathway, fermentation and starch metabolism), and $17.7 \%$ of DEPs participated in stress/defense regarding redox homeostasis and removal of aldehydes. Conclusion Multiple metabolic pathways in the developing caryopsis were affected by $\mathrm{N}$ topdressing, especially the mitochondrial respiration, sucrose-to-starch metabolism and $\mathrm{N}$ metabolism. The insufficient supply of ATP energy as a result of the significantly lowered mitochondrial respiration induced by $\mathrm{N}+$ regime inhibits the sucrose-to-starch metabolism and starch biosynthesis in developing grains, and is strongly responsible for grain chalk formation under $\mathrm{N}$ topdressing.

\section{Background}

Grain chalkiness is used as a vital factor for grading and determining the price in the market and consumption of rice grain. Compared with vitreous ones, chalky grains exhibit loosely packed starch granules and larger air spaces in endosperm cells [1,2]. This chalk in endosperm consequently leads to an inferior palatability for changes in amylose content, protein content and chain length distributions of amylopectin $[3,4]$. Now, the reduction in grain quality due to the occurrence of chalkiness has become a serious problem in many rice producing areas of the world [5]. Thus, it is very important to achieve high grain quality by lowering the chalky occurrence of rice cultivars.

Except for the genotypic variation among different rice cultivars, grain chalkiness is readily affected by environmental factors, particularly the high temperature $(\mathrm{HT})$ and chemical nitrogen $(\mathrm{N})$ fertilizer application at the late growth stage. HT during grain-filling period accelerates the growth rate of endosperm and causes grain chalkiness [6, 7]. Intensive investigation revealed that HT exposure impaired the biosynthesis and deposition of starch in rice grains as a result of HT-induced down-regulation of various genes involving in starch biosynthesis [8-10]. Nevertheless, little information was available on the metabolic mechanism underlying the effect of $\mathrm{N}$ fertilizer on grain chalk occurrence. 
The element $\mathrm{N}$ is a vital constituent of nucleotides and proteins for plant metabolism, and its application is one important agronomic practice determining crop yield and quality. To achieve high yields, more $\mathrm{N}$ fertilizer than the maximum of crop required was applied to rice at the expense of grain quality.

Sveral studies found that the percentage of chalky grains was decreased with increasing $\mathrm{N}$ rates, even if $\mathrm{N}$ topdressing [11-13]. But some other reports revealed that the chalky grain rate increased with the increase of $\mathrm{N}$ applied amount at the late growth stage $[14,15]$. The topic on chalkiness characters response to $\mathrm{N}$ fertilization is discussed controversially. For the absence of representative resources, studies being performed were confined to analyze chalky characters at maturity, which hardly explored the molecular basis for chalkiness formation during grain-filling stage.

Grain chalkiness is a complicated quantitative trait that is controlled by many genes. To date, many quantitative trait loci (QTLs) genetically related to grain chalkiness trait have been detected by various groups [16-18], and the phenotypic variation of grain chalky occurrence among different rice cultivars could be partly explained by these QTLs. For instance, introducing the gs9 allele into elite rice cultivars significantly lowered grain chalky occurrence, and markedly enhanced grain appearance quality [19]. However, only few QTLs have been isolated and functionally identified, and the regulation mechanism remains largely unknown thus far.

To clarify the metabolism of grain chalky occurrence in response to $\mathrm{N}$ topdressing, a comparative proteomic analysis of rice caryopses by iTRAQ technology was performed to investigate the expression pattern of many functional proteins responses to $\mathrm{N}$ treatment. A total of 198 proteins involved diverse but delicately regulated pathways were identified. Such result shall provide a helpful knowledge for our understanding on the metabolic mechanism of grain chalky occurrence caused by $\mathrm{N}$ topdressing during grain filling.

\section{Methods}

\section{Plant materials}

Experiments were carried out in cement plots at the agronomy research farm of Anhui Academy of Agricultural Sciences $\left(31^{\circ} 86^{\prime} \mathrm{N}, 117^{\circ} 27^{\prime} \mathrm{E}\right)$, Hefei city, Anhui province, China in 2016 and 2017. An indica rice cultivar OM052 was used in this study. OM052 was explored by Rice Research Institute, Anhui Academy of Agricultural Sciences, Anhui province, China. Interestingly, it has a percentage of chalky grains over $90 \%$ with the area of endosperm chalkiness exceed $40 \%$ (Additional file 1: Figure S1). This rice cultivar is thus suitable to explore the metabolism variations of chalky grains response to $\mathrm{N}$ topdressing at the molecular and biochemical levels during grain-filling stage. The rainfall and average temperature during growing seasons were shown in Additional file 2 (Figure S2). The average daily temperatures during grain-filling stages (form 10 August to 5 October) were $26.1^{\circ} \mathrm{C}$ in 2016 and $24.4{ }^{\circ} \mathrm{C}$ in 2017, respectively. The monthly rainfalls in August and September were $60.4 \mathrm{~mm}$ and $128.6 \mathrm{~mm}$ in $2016,243.3 \mathrm{~mm}$ and $111.6 \mathrm{~mm}$ in 2017, respectively. Soil samples from the upper $20 \mathrm{~cm}$ layer were collected for analysis of properties before the rice was transplanted in the first year. The soil had a clay 
loam texture with $10.9 \mathrm{~g} \mathrm{~kg}^{-1}$ organic matter, $0.68 \mathrm{~g} \mathrm{~kg}^{-1}$ total $\mathrm{N}, 7.9 \mathrm{mg} \mathrm{kg}^{-1}$ available phosphorus $(\mathrm{P})$ and $167.3 \mathrm{~g} \mathrm{~kg}^{-1}$ available potassium (K).

Experimental design

The design was a randomized design with three replicates in both tested years. Each plot was $8.0 \mathrm{~m} \times 1.5$ $\mathrm{m}$. Two $\mathrm{N}$ fertilizer topdressing treatments were conducted at the panicle initiation stage: (1) N0, without $\mathrm{N}$ topdressing to rice; (2) $\mathrm{N}+, 108 \mathrm{~kg} \mathrm{~N} \mathrm{ha}^{-1}$ applied to rice. In all treatments, $\mathrm{N}$ fertilizer in the form of urea was applied with $135 \mathrm{~kg} \mathrm{~N} \mathrm{ha}^{-1}$ as basal fertilizer, $27 \mathrm{~kg} \mathrm{~N} \mathrm{ha}^{-1}$ at 7 days after transplanting, and the $\mathrm{N}$ dose applied at panicle initiation according to the experimental treatments. A recommended dose of 90 $\mathrm{kg} \mathrm{Pha}^{-1}$ (Di ammonium phosphate) and half of $180 \mathrm{~kg} \mathrm{~K} \mathrm{ha}^{-1}$ (Potassium sulfate) was applied as basal fertilizer uniformly to all plots along with control. The rest of $\mathrm{K}$ fertilizer was applied at the panicle initiation.

Rice seeds were sown on 13 May 2016, and 15 May 2017. The seedlings were transplanted on 1 June 2016 and 3 June 2017, and harvested on 2 October and 5 October in 2016 and 2017, respectively. The rice seedling was transplanted to the plots at a density of 25.1 hills $\mathrm{m}^{-2}$ (a spacing of $13.3 \mathrm{~cm} \times 30.0 \mathrm{~cm}$ ) with two plants per hill. Weeds, pests and diseases were intensively controlled to avoid yield loss.

Sampling and measurement

A total of 300 panicles with uniformity anthesis day were randomly selected and tagged at the heading stage, and part of the tagged panicles (20 tagged panicles) were sampled at 15 days after flowering (DAF). As the method of You et al. [20], the primary branches in the middle part of the panicle were collected, and immediately frozen in liquid $\mathrm{N}$ and stored at $-80^{\circ} \mathrm{C}$ for protein extraction. At maturity, about 280 tagged panicles with similar maturity were harvested. These samples were naturally dried, and stored at room temperature for 3 months.

Scanning electron microscopy (SEM) and physicochemical composition analysis

For observation of cross-sections of endosperm, dried rice seeds were cut across the short axis with a razor blade. The surface was sputter coated with gold in vacuum and observed by SEM (Hitachi S$3000 \mathrm{~N}$ ) at an accelerating voltage of $10 \mathrm{kV}$. Grain chalkiness traits were determined by WSeen's rice appearance quality detection system (Model SC-E) according to the industrial standard of Agriculture Ministry (NYT 2334-2013). Length/width (L/W) ratio was measured as the ratio of length to width using digital vernier calipers for an average of ten seeds. For chemical composition analysis, rice seeds were ground to pass a 100-mesh sieve. Amylose content was determined using iodine colorimetry via alkaline steeping. Protein fractions for albumins, globulins, prolamins and glutelins were extracted by water, $10 \% \mathrm{NaCl}, 55 \% \mathrm{n}$-propanol and Biuret reagent in sequence, and analyzed as described by Liu et al. [21]. Total protein content was calculated as the sum of the four fractions with three replicates.

Protein extraction 
Total proteins from endosperm tissue were extracted as previously described [22] with minor modifications. About $0.1 \mathrm{~g}$ samples were homogenized in liquid $\mathrm{N}$, and added into $2 \mathrm{~mL}$ lysis buffer (30\% sucrose, $0.5 \mathrm{M}$ Tris- $\mathrm{HCl}, 50 \mathrm{mM}$ EDTA, $20 \mathrm{mM}$ DTT, $0.1 \mathrm{M} \mathrm{KCl}, 2 \%$ SDS, 1x Protease Inhibitor Cocktail (Roche Ltd. Basel, Switzerland)). Cell lysis was performed by sonication on ice for $1 \mathrm{~h}$, followed by centrifugation at $4,000 \mathrm{~g}$ for $30 \mathrm{~min}$ at $4{ }^{\circ} \mathrm{Cin}$ a refrigerated high-speed centrifuge. Equal volumes of Trissaturated phenol $(\mathrm{pH} 7.5)$ were added and the resulting mixture was thoroughly vortexed for $30 \mathrm{~min}$ at $4^{\circ} \mathrm{C}$ and centrifuged at $4,000 \mathrm{~g}$ for $15 \mathrm{~min}$ at $4{ }^{\circ} \mathrm{C}$ for phase separation. Then, the organic phase was collected and transferred into a new Eppendorf tube. The extraction step was repeated once. The combined organic phase was precipitated using five volumes of prechilled acetone at $-20^{\circ} \mathrm{C}$ overnight, followed by centrifugation at $10000 \mathrm{~g}$ for $10 \mathrm{~min}$ at $4{ }^{\circ} \mathrm{C}$. The precipitate was washed twice with cold acetone and then resuspended with $2 \%$ SDS.

Protein digestion and iTRAQ labeling

Proteins were redissolved in $500 \mathrm{mM}$ TEAB (triethylammonium bicarbonate). Protein concentration of the supernatant was determined by using the BCA protein assay, and $100 \mu \mathrm{g}$ proteins were transferred into a new tube and adjusted to a final volume of $100 \mu \mathrm{L}$ with $8 \mathrm{M}$ ureas. $11 \mu \mathrm{L}$ of $1 \mathrm{M}$ DTT was added, and incubated at $37^{\circ} \mathrm{C}$ for $1 \mathrm{~h}$. The solution was then transferred into $10 \mathrm{~K}$ ultrafiltration tube to centrifuged by adding $100 \mathrm{Mm}$ TEAB for three times to remove urea. Subsequently, $120 \mu \mathrm{L}$ of $55 \mathrm{mM}$ iodoacetamide was added to the sample and incubated for 20 min protected from light at room temperature. Then proteins were digested with sequence-grade modified trypsin (Promega, Madison, WI). The resultant peptide mixture was labeled using iTRAQ 8-plex reagent following the manufacture's instruction (AB Sciex Inc., USA). The N0 samples were labeled 115,116 and 117; N+ samples were labeled 118, 119, and 121, respectively. All labeled peptide samples were then pooled, and lyophilized in a vacuum concentrator for further identification.

Nano-high performance liquid chromatogra with tandem mass spectrometry (nano-HPLC-MS/MS) analysis

The peptide mixture was redissovled in buffer $\mathrm{A}(20 \mathrm{mM}$ ammonium formate in water, $\mathrm{pH} 10.0$, adjusted with ammonium hydroxide), and fractionated by high $\mathrm{pH}$ separation using Ultimate 3000 system (ThermoFisher scientific, MA, USA) with a reverse phase column (XBridge C18 column, $4.6 \mathrm{~mm} \times 250 \mathrm{~mm}$, $5 \mu \mathrm{m}$; Waters Corporation, MA, USA) at $1 \mathrm{~mL} / \mathrm{min}$ using a linear binary gradient starting from $5 \%$ buffer $B$ to $45 \%$ buffer $\mathrm{B}(20 \mathrm{mM}$ ammonium formate in $80 \% \mathrm{ACN}, \mathrm{pH} 10.0$, adjusted with ammonium hydroxide) in $40 \mathrm{~min}$. Twelve fractions were collected, and each was dried in a vacuum concentrator for next step.

The dried peptide samples were resuspended in $30 \mu \mathrm{L}$ solvent $\mathrm{C}$ (water with $0.1 \%$ formic acid) and separated by nano-LC. The UPLC system (NanoACQuity, Waters) was used with a Q-Exactive mass spectrometer (Thermo Fisher Scientific, MA, USA). Samples were loaded on a trap column (Thermo Scientific Acclaim PepMap C18, $100 \mu \mathrm{m} \times 2 \mathrm{~cm}$ ), with a flow of $10 \mu \mathrm{L} / \mathrm{min}$ for $3 \mathrm{~min}$, and then separated on an analytical column (Acclaim PepMap C18, $75 \mu \mathrm{m} \times 15 \mathrm{~cm}$ ) with a $90 \mathrm{~min}$ linear gradient from $5 \%$ to $55 \%$ D (ACN with $0.1 \%$ formic acid). The column was re-equilibrated at initial conditions for $10 \mathrm{~min}$, and 
the flow rate was maintained at a $300 \mathrm{~nL} /$ min with spray voltage of $2.0 \mathrm{kV}$. The mass spectrometer was run under data dependent acquisition mode, automatically switched between MS and MS/MS mode. Dynamic exclusion time was $25 \mathrm{~s}$.

Bioinformatics analysis

MS/MS spectra were processed by PEAKS Studio version 8.5 (Bioinformatics Solutions Inc., Waterloo, Canada) against Oryza sativa database of UniProt (ver. 201712, 37383 entries). During the search, proteins were identified with a fragment ion mass tolerance of $0.05 \mathrm{Da}$, parent ion tolerance of $7.0 \mathrm{ppm}$, fixed modification of carbamidomethylation, variable modifications of oxidation at Met and iTRAQ 8-plex at Tyr. Peptides were filter by $1 \%$ FDR and 1 unique. ANOVA was used for peptide and protein abundance calculation. Normalization was performed on averaging the abundance of all peptides, and medians were used for averaging. Differential expressed proteins (DEPs) were filtered if their fold change were over 1.5 and contained at least 2 unique peptides with $P$-value $<0.05$. These obtained proteins were classified using the Gene Ontology (GO) database and Kyoto Encyclopedia of Genes and Genomes (KEGG) database.

Quantitative real-time PCR (qRT-PCR) analysis

Total RNA from endosperm sampled was extracted using an RNAiso Plus kit (TaKaRa, Dalian, China), and transcribed to cDNA with Transcriptor First Strand cDNA Synthesis Kit (Roche, Mannheim, Germany) according to the manufacturer's instructions. qRT-PCR was done using a SYBR Green Master Mix kit (Vazyme, Nanjing, China). Sequences of primers are listed in Additional file 5 (Table S3). PCR products were separated by electrophoresis in $1.0 \%$ agarose gels, stained with GelStain (TransGen Biotech, Beijing, China), and visualized using Ultraviolet transmittance instrument (HoferMV-25, Amersham Pharmacia).

Statistical analysis

Microsoft Excel 2007 and Origin 2018 software were used for data processing. The variance analysis was performed by Duncan's new multiple-range test at the 5\% probability level in SPSS 20.0 statistical software.

\section{Results}

Effect of $\mathrm{N}$ topdressing on grain chalkiness

$\mathrm{N}+$ treatment significantly increased the area of endosperm chalkiness (AEC) and the degree of grain chalkiness (DGC), although the insignificant difference in the percentage of chalky grains (PCG) was observed between N+ and NO (Fig. 1). Compared with N0, the AEC and DGC were increased by $14.88 \%$ and $13.65 \%$ by $\mathrm{N}+$ in $2016,12.47 \%$ and $13.60 \%$ in 2017 , respectively. Across different $\mathrm{N}$ treatments, the average PCG, AEC and DGC displayed over $95 \%, 44 \%$ and $43 \%$ in both years, respectively. Therefore, OM052 represents an idea genetic material with relatively stabilized genetic background suitable for exploring the molecular mechanism of chalkiness formation under $\mathrm{N}$ topdressing. 
Effects of $\mathrm{N}$ topdressing on physiochemical compositions in rice

As shown in Table 1, N+ didn't differ from N0 in grain length/width (L/W). Remarkably, N+ decreased grain amylose content and increased grain protein content, regardless of experimental years (2016 and 2017). Further analysis of protein components reveled that the glutelin was significantly increased by $\mathrm{N}+$ in comparison with NO treatment, and albumin, globulin and prolamin slightly fluctuated between N treatments. The weight of rice grains subjected to $\mathrm{N}+$ exhibited a decrease compared to that of the control.

$\mathrm{N}$ topdressing effect on endosperm structure of rice grains

A marked difference in the distribution of starch granules and the amount of protein bodies was observed between chalky part and translucent one by using SEM images (Fig. 2), with the loosely packed starch granules in chalky part. In contrast, the rice endosperm sampled from $\mathrm{N}+$ was observed to have relatively more abundant numbers of protein bodies than that from NO. This finding implied that the effect of $\mathrm{N}$ topdressing on grain chalky appearance might be closely associated with the formation of starch granules and protein bodies in filling rice endosperms.

Effect of $\mathrm{N}$ topdressing on the expression of proteins

To clarify the underlying molecular basis for chalky endosperm formation under $\mathrm{N}$ topdressing, an iTRAQbased quantitative proteomic analysis was used to compare overall differences in the proteomes of the two experiment groups ( $\mathrm{N} 0$ and $\mathrm{N}+$ ). A total of 1154 proteins were identified with FDR $<1 \%$, and their changes in protein abundance were assessed (Additional file 3: Table S1). Prior to bioinformatic analysis of DEPs, principal component analysis (PCA) was performed to determine the global relationship in the protein data. The results showed that the first two principal components could explain $76.67 \%$ of the variation (Fig. 3a). Additionally, we also separate the replicates from each treatment into two discrete groups (Fig. 3b), which is a further indication for the reliable data.

Functional classification of differentially expressed proteins

According to a 1.5 -fold change or a 0.67 -fold, a total of 198 proteins, together with the number of unique peptides $\geq 2$, showed differential expression between $\mathrm{N}+$ and $\mathrm{N} 0$, including 9 up-regulated proteins and 189 down-regulated proteins for the rice plants imposed to $\mathrm{N}+$ treatment (Table 2, 3). These DEPs could be divided into 12 major categories, in term of their biological functions and metabolic pathways (Fig.4a). Among them, the largest category, accounting for $22.7 \%$ of total DEPs, was participated in protein synthesis, folding, degradation and storage. The second category, about $21.7 \%$ of total DEPs, was the differential proteins that participated in carbohydrate (C) metabolism, including glycolysis, tricarboxylic acid (TCA) cycle, pentose phosphate pathway (PPP), fermentation and starch metabolism (Fig.5). The third category, accounting for $17.7 \%$ of the total DEPs, belonged to stress/defense regarding redox homeostasis and removal of aldehydes. Besides, 17 proteins ( $8.6 \%$ of total DEPs) were thought to function in grain amino acid synthesis and catabolism. 
The 9 up-regulated proteins induced by $\mathrm{N}+$ treatment contributed mainly to storage protein (glutelin) biosynthesis and stress/defense. Whereas, the 189 down-regulated proteins were closely related to $\mathrm{C}$ metabolism, protein metabolism, amino acid metabolism, stress/defense, photosynthesis and electron transfer, lipid metabolism, cell growth/division, material transport, signal transduction, energy metabolism, and unknown proteins with yet unidentified function or no detectable homology in the database compared with N0 treatment. Of these 198 proteins identified, $70.7 \%$ of the DEPs were associated with gain $\mathrm{C}$ metabolism, $\mathrm{N}$ metabolism and stress/defense with redox homeostasis (Fig. 5,6$)$. And in $\mathrm{C}$ metabolism and protein metabolism functions, the number of DEPs related to central carbon metabolism, starch metabolism and protein synthesis were more than other protein functions (Fig. 4 b, c). This result suggests that $\mathrm{N}+$ impaired the sucrose-to-starch translocation and $\mathrm{N}$ metabolism, which was ascribed to insufficient carbohydrates and energy supply in chalky endosperms.

qRT-PCR analysis

To monitor the expression pattern of the proteins identified by iTRAQ, twelve proteins were selected for qRT-PCR analysis, including granule-bound starch synthase I (A2Y8X2), soluble starch synthase I (A2Y9M4), UDP-glucose pyrophosphorylase (A3QQQ3), ADP-glucose pyrophosphorylase large subunit(P93430) and small subunit (A2YU91), disulfide isomerase (A2ZCE6), glucose-6-phosphate translocator (B8BBE6), aspartate aminotransferase(B8AEH4), glutamine synthetase (B8AQE3), glutelin (B8A9T8), cytosolic orthophosphate dikinase (B8AYC1) and allergen (A2YJG5) (Additional file 4: Table S2). Transcript levels of the genes and abundance of the corresponding proteins displayed similar trends (Fig. 7), further verifying the reliability of the iTRAQ technology.

\section{Discussion}

In this research, an iTRAQ-based technology combined with a typical rice material with chalkiness rate over $90 \%$ was used to clarify the mechanism behind the occurrence of chalky grains as influenced by $\mathrm{N}$ topdressing. We discovered that the formation of endosperm chalk caused by $\mathrm{N}$ topdressing involves the coordinated regulation of multiple metabolic pathways, especially mitochondrial respiration, sucrose-tostarch metabolism and $\mathrm{N}$ metabolism.

Relationship of respiratory metabolism and starch synthesis in developmental endosperms with chalky appearance induced by $\mathrm{N}$ topdressing

C metabolism primarily includes three key metabolic pathways (glycolysis, PPP and TCA cycle), which provides the energy and material for transformation and synthesis of metabolites [23, 24]. Completion of the prominent glycolysis and TCA cycle require an adequate supply of ATP and the synthesis of cellular components essential for the cell enlargement and starch synthesis [20, 25]. In this study, the expression abundance of most identified proteins related to glycolysis and TCA cycle was much lower at N+ than at N0 (Table 3; Fig. 5). Among them, the enzymes of phosphofructokinase (PFK) and pyruvate kinase (PK) are rate-limiting enzymes of glycolysis and catalyze the important exergonic steps. Their reduced expression levels drastically impaired the progress of glycolysis, and cellular respiration. Concurrently, the 
TCA cycle completed the oxidation of glucose was largely restrained supported by the down-regulated proteins involved, such as malate dehydrogenase (MDH), succinate dehydrogenase (SDH), aconitate (ACO) cytoplasmic and so on. Further, proteins related to the pathway of PPP like glucose and ribitol dehydrogenase homolog 1, triosephosphate isomerase (TPI) were down-regulated by $\mathrm{N}+$, which can greatly enhance plant adaptability in the stress by cooperating with glycolysis and TCA cycle. Above results indicate that the inhibited respiration with slowed glycolysis, TCA cycle and the reduced PPP in grains subjected to $\mathrm{N}$ topdressing might reduce the endosperm cell growth and synthesis of storage substances.

Additionally, we will consider the process of fermentation that occurs in the absence of oxygen, whereby pyruvate is reduced with concomitant oxidization of NADH to NAD+, and the generating of ATP anaerobically. The fermentation is hence very important for keeping an appropriate ATP level for starch synthesis when oxygen is insufficient [25]. In our study, three key enzymes in alcohol fermentation pathway, pyruvate decarboxylase 2 (PDC2), alcohol dehydrogenase 1 and 2, were down-regulated by $\mathrm{N}+$, implying a detrimental effect of restricted fermentation on the synthesis of starch and protein.

In brief, proteomics reveal that a weakened central carbon metabolism and alcoholic fermentation, and a switch between them affected the synthesis and deposition of starch due to the short supply of ATP and donors, and finally increasing grain chalk when $\mathrm{N}$ topdressing. However, to date, there have been few researches in which starch synthesis in cereal endosperm has been considered with regard to energy requirements of storage product deposition. Thus, to explore the metabolic mechanism behind the formation of grain chalkiness thoroughly, more emphasis should be placed on interactions between starch synthesis and respiration during endosperm development. Whilst it is not overlooked that the effect of restraining mitochondrial respiration on the partition of sucrose into starch and protein synthesis.

Defects in starch synthesis under $\mathrm{N}$ topdressing related to grain chalkiness

Starch is a predominant storage substance in rice grain, occupying over $80 \%$ of the total dry mass, its insufficient deposition is responsible for rice gain chalkiness [1,3]. During grain filling, starch is synthesized via enzymatic reactions after sucrose degradation [26, 27]. In this study, proteins involved in sucrose degradation and starch biosynthesis, uridine diphosphate glucose pyrophosphorylase (UGPGase), adenosine diphosphate glucose pyrophosphorylase large subunit and small subunit

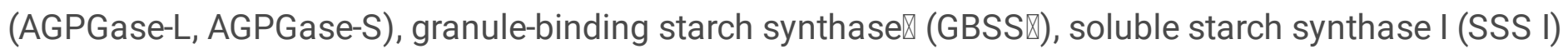
and pullulanase (PUL), were down-regulated by $\mathrm{N}+$ (Fig.5). The inactivation of the UGPase1 gene or disturbed starch synthesis could cause endosperm chalkiness in rice [28, 3]. In our study, the reduced UDPGase along with the repressed starch biosynthesis-related enzymes indicate that sucrose-to-starch metabolism was restrained, which might involve in the response of chalky endosperm to $\mathrm{N}$ topdressing. Moreover, we also found proteins associated with transport of metabolites for starch synthesis were also down-regulated by $\mathrm{N}+$, comprising glucose-6-phosphate translocator (GPT) and plastidic glucose transporter 4(PGIc), adenine nucleotide transporter (ANT). Their lower activities greatly slowed down 
metabolites import from the cytosol to support starch biosynthesis in the endosperm cell, and might partially explain the formation of grain chalk under $\mathrm{N}+$.

Besides, recent researches have awarded the role of starch hydrolysis in the occurrence of grain chalkiness, which was powerfully evidenced by micro-pores on the surface of the compound granules [29, 30]. By contrast, Ishimaru et al. [31] indicted that starch degradation by a-amylase was not the cause of the formation of chalky grain because a-amylase mRNA was not detected. In this study, alphaglucosidase (AGS) involved in starch hydrolysis, was down-regulated in grains grown at N+ as compared with those at N0, while three allergenic informs that predicted connected with the inhibition of starch hydrolysis were notably up-regulated by $\mathrm{N}+$. This promotes starch component synthesis and accumulation in the developing rice grains, and confirms the relation between starch hydrolysis and grain chalky appearance.

Low abundances of proteins involved in energy metabolism

We also identified proteins involved in the regulation of energy metabolism, viz. ATP synthase subunit, ATPase and adenosine kinase (ADK) 2. Of which, particular ADK plays a key role in maintains energy homeostasis of ATP, AMP and ADP, and in the regulation of adenosine levels in starch and nucleic acid metabolites [32]. Previous study exhibited that increasing adenosine in the upstream of starch metabolism pathway could result in an increase in the starch biosynthesis [33]. Decreasing ADK activity was beneficial to raise adenosine sink and ATP accumulation in plant, and consequently raising carbohydrate accumulation including starch [34]. Our proteomic study showed that the three proteins were down-regulated by $\mathrm{N}+$. This finding suggests that the broken energy homeostasis and the regulation of intracellular and extracellular levels of adenosine are closely with grain chalk appearance and the normal seed development. That should be further investigated the importance of energy metabolism and ADK role in regulating primary metabolism during grain-filling when studies on grain chalkiness.

Restrained protein metabolism (protein synthesis, folding, and degradation) and varied storage protein stimulated endosperm chalkiness exposed to $\mathrm{N}$ topdressing

Recent work found the potential role of protein biosynthesis or $\mathrm{N}$ metabolism in the occurrence of grain chalkiness, which ever has been underestimated in studies on rice quality. For instance, an abnormal expression of binding protein (BiP) negatively affected the accumulation of seed storage proteins, and produced an opaque phenotype seed with a rice floury endosperm [35]. Protein synthesis is one most complex biological processes containing translation initiation and elongation, newly synthesized polypeptides folding and assembly. In this study, proteins related to protein synthesis, degradation and storage as well as amino acid metabolism were found to be differentially expressed between $\mathrm{N}+$ and $\mathrm{N} 0$, implying the role of proteins in grain chalkiness formation.

Ribosome subunits like a small 40S subunit and a large 60S subunit involve in the cellular process of translation. Acidic ribosomal phosphorylation proteins (P proteins) located on 605 subunit, including P0, $\mathrm{P} 1$ and $\mathrm{P} 2$, are unique among ribosomal constituents and take part in interactions with elongation factors 
during the course of protein synthesis [36]. In this study, the total of 15 down-regulated proteins regarding translation initiation and elongation were detected in grains from $\mathrm{N}+$, including $40 \mathrm{~S}$ ribosomal (S14, S12, S20-1-like, S3-3), 60S ribosomal (L14-1, L7-2, L36-3-like, L21), 60S acidic ribosomal (P2B, P3, P2A, P1) and even translation initiation factor $5 \mathrm{~A}$ (elF-5A), mitochondrial translation elongation factor Tu, elongation factor 1 -beta. This result indicates that the restrained protein translation initiation and elongation in developing grains exposed to $\mathrm{N}$ topdressing contributed to the formation of grain chalkiness.

To fulfill their biological roles, proteins must fold into precise 3-dimensional shape that is governed by proteins known as molecular chaperones. In endoplasmic reticulum (ER), protein disulfide isomerase (PDI), 70-kD heat shock proteins (Hsp70s) primarily are main molecular chaperones assisting proper polypeptide folding and preventing unfolded proteins aggregation [37]. The failed expression of protein disulphide isomerase-like 1-1 (PDIL1-1) led to an abnormal accumulation of glutelin precursor in the endosperm [38]. Further, small grains with a floury endosperm or a chalky phenotype with lower protein content was observed in a mutant T3612 deleting PDIL1-1 or in grains repressing PDIL1-1 expression [39, 40]. A positive correlation was also observed between small Hsps and the appearance of chalky kernels under HT [41]. In this study, molecular chaperones, PDI, PDIL1-4, stromal 70 kDa heat shock-related chloroplastic, etc., were down-regulated by $\mathrm{N}+$, while proteins related to glutelins and their precursors were up-regulated. The results indicate that the reduced level of PDI and other chaperones by $\mathrm{N}+$ is most likely to alter storage protein deposition during grain filling, which might relate to grain chalk. We hence suggest that the interacts of ER chaperones with metabolic system for protein synthesis, and the relationships between molecular chaperones and accumulation of seed storage proteins should be clarified in the coming studies on chalky grains response to $\mathrm{N}$ fertilizer.

Regulating protein degradation plays a key role during the development of organisms. The ubiquitinproteasome pathway is one main mechanism for proteolysis in eukaryotes, which promotes covalent attachment of ubiquitin to protein substrates through sequential action of three enzymes called ubiquitinactivating enzyme (E1), ubiquitin-conjugating enzyme (E2), and ubiquitin-protein ligase (E3) [42]. Most ubiquitinated proteins are then recognized and degraded by $26 \mathrm{~S}$ proteasome [43]. In this paper, the expressed abundances of protein degradation-related proteins like E3 ubiquitin-ligase RGLG2-like, proteasome subunit alpha type, proteasome subunit beta type-2, proteasome subunit alpha type-7-A-like, etc., were lower in grains under $\mathrm{N}+$ than those under $\mathrm{N} 0$. This result implied the involvement of proteolysis in rice grain chalkiness response to $\mathrm{N}$ topdressing, and the decreased proteolysis during the grain-filling stage by $\mathrm{N}+$ hinder seed endosperm development, causing chalk texture. The relations of proteolysis and the occurrence of grain chalky appearance were also found by Lin et al. [44].

Storage proteins are specifically synthesized during seed maturation, and deposited into protein bodies via ER lumen. Of which, the staple storage protein glutelin are synthesized as a 57-kD proglutelin precursor $[45,46]$. Previous study demonstrated that the differentially expression of storage proteins with up-regulated at early filling stage and down-regulated at maturation might partially explain the increase of rice grain chalk under $\mathrm{HT}$ [47]. A reduction of one or a few seed storage proteins was compensated for 
by increases in other storage proteins at both the mRNA and protein levels [48]. In this paper, proteins defined as glutelin were differentially enhanced by $\mathrm{N}+$, such as glutelin and its precursor, cupin family expressed and globulin-1 $\mathrm{S}$ allele-like. This finding implied that the composition and accumulation configuration of seed storage protein was changed by $\mathrm{N}$ topdressing, and the increased glutelin-related expression abundance may deteriorate endosperm chalkiness under $\mathrm{N}$ topdressing. We hence suspect that the interactions and changes of storage proteins composition in developing rice grains are involved in regulating the formation of endosperm chalk under $\mathrm{N}$ topdressing, though the specific regulatory mechanism involved requires further study.

Ornithine cycle involved in the response of chalky grain to $\mathrm{N}$ topdressing

Ornithine cycle is a cyclic enzymatic process consisting of consecutive transformations of ornithine and leading to the synthesis of urea, which is an important pathway of $\mathrm{N}$ metabolism in plant $[49,50]$. The yielding urea will be decomposed and involved in amino acids synthesis by glutamine synthetaseglutamate synthase (GS-GOGAT) pathway [51]. In this study, proteins consisting of argininase (ARG) mitochondrial, argininosuccinate (ASS) chloroplastic and ornithine transcarbamoylase (OTC) involved in ornithine cycle were down-regulated by $\mathrm{N}+$ (Fig.5). The date from our study showed that the weakened ornithine cycle will cut down the hydrolysis of arginine to ornithine and urea, inhibits the reuse of $\mathrm{N}$ and rice caryopsis development, and is partly responsible for the increase of endosperm chalk under $\mathrm{N}+$. But the defined role of ornithine cycle in the response of grain chalkiness to $\mathrm{N}$ application needs deserves to be clarified.

Additionally, transamination in plant is a crucial process of $\mathrm{N}$ metabolism that

is accomplished by a bevy of transaminase enzymes, particularly aspartate amino transferase (GOT) and alanine amino transferase (GPT) [52]. Inorganic $\mathrm{N}$ is converted to amino acids by catalysis of the two transaminases, thereby providing a variety of amino acid donors for grain proteins synthesis and metabolism [53]. Our proteomic date revealed that the abundances of GOT and GPT in filling grains exposed to $\mathrm{N}+$ treatment were significant lower than those exposed to N0 treatment. Also, GS that takes part in GS-GOGAT pathway was detected down-regulated by N+ treatment in this study. This finding indicates that the reduced rate of $\mathrm{N}$ assimilation induced by $\mathrm{N}$ topdressing resulted in a poor $\mathrm{N}$ metabolism during the critical grain-filling stage, which may be closely with the occurrence of chalk in grains.

Toward a bettering understanding for the mechanism underlying grain chalk formation induced by $\mathrm{N}$ fertilizer in terms of $\mathrm{C} \times \mathrm{N}$ metabolism interaction

$\mathrm{C}$ and $\mathrm{N}$ metabolism are two major physiological processes during grain filling, and their end-products starch and protein are main biochemical components of rice grains [54]. As well as the deposition of starch, this is also the period in which storage protein is deposited. Both starch and proteins are thus equally significant for grain quality, such as the translucent of seeds appearance. However, numerous studies in regard to grain chalkiness have focused on $\mathrm{C}$ metabolism and its outcome starch, involving its synthesis and degradation [9, 31]. Our previous work showed that the insufficient deposition of protein 
bodies, which do not completely fill the air spaces between starch granules, might be explain the chalk occurrence [1]. In this study, comprehensive analysis showed that over one third of DEPs closely associated with $\mathrm{N}$ metabolism, comprising protein synthesis, folding, degradation and storage as well as amino acid metabolism. Particularly, the changes in the abundances of storage protein glutelin, the molecular chaperones of PDI, Hsp70s, proteasome, and urea cycle-related proteins, indicate the potential role of $\mathrm{N}$ metabolism in the formation of grain chalkiness induced by $\mathrm{N}$ topdressing. Thus, to clarify the underlying mechanism of grain chalk occurrence, studies referring to grain $\mathrm{N}$ metabolism and its outcome protein accumulation are expected to pay more attention. Most importantly, studies concerning interactions between $\mathrm{C}$ and $\mathrm{N}$ metabolism, and the partition towards of the products of respiratory metabolism during critical grain filling stage are indispensable in terms of the substantial relationship between them.

\section{Conclusions}

Ripening under $\mathrm{N}$ topdressing led to a serious chalky texture in grains. These grains subjected to $\mathrm{N}$ topdressing showed a decreased amylose content but increased protein content as compared with the control. Proteomic date revealed that multiple metabolic pathways in the developing caryopsis were affected by $\mathrm{N}$ fertilizer topdressing, especially mitochondrial respiration, sucrose-to-starch metabolism and $\mathrm{N}$ metabolism. The insufficient supply of ATP energy as a result of the significantly lowered mitochondrial respiration induced by $\mathrm{N}+$ regime had an inhibitory impact on the sucrose-to-starch metabolism and starch biosynthesis in developing grains, which is strongly responsible for grain chalk formation under $\mathrm{N}$ topdressing. Restrained protein synthesis and degradation as well as varied storage protein might stimulate endosperm chalk when $\mathrm{N}$ topdressing. An integrated method using a combination of omics platforms like metabolomic and transcriptomic will be required to understand the mechanism comprehensively.

\section{Abbreviations}

iTRAQ: Isobaric tags for relative and absolute quantification; N: Nitrogen; DAF: Days after flowering; DEPs, Differentially expressed proteins; QTLs: Quantitative trait loci; HT: High temperature; HPLC-MS/MS: High performance liquid chromatography with tandem mass spectrometry; SEM: Scanning electron microscopy; 2D: Two dimensional; UPLC: Ultra performance liquid chromatography; GO: Gene Ontology; KEGG: Kyoto Encyclopedia of Genes and Genomes; qRT-PCR: Quantitative real-time PCR; ER : Endoplasmic reticulum; ADK: Adenosine kinase; BiP: Binding protein; P proteins: Phosphorylation proteins; PDI: Protein disulfide isomerase; Hsp70s: 70-kD heat shock proteins; PDIL: Protein disulphide isomerase-like; E1: Ubiquitin-activating enzyme; E2: Ubiquitin-conjugating enzyme; E3: Ubiquitin-protein ligase; C: Carbohydrate; P: Phosphorus; K: Potassium; L/W: Length/width; DGC: Degree of grain chalkiness; AEC: Area of endosperm chalkiness; PCG: Percentage of chalky grains; PCA: Principal component analysis; TCA: Tricarboxylic acid; PPP: Pentose phosphate pathway; PFK:

Phosphofructokinase; PK: Pyruvate kinase; MDH: Malate dehydrogenase; SDH: Succinate dehydrogenase; 
ACO: Aconitate; TPI: Triosephosphate isomerase; PDC2: Pyruvate decarboxylase 2; UGPGase: Uridine diphosphate glucose pyrophosphorylase; AGPGase-L: Adenosine diphosphate glucose pyrophosphorylase large subunit; AGPGase-S: Adenosine diphosphate glucose pyrophosphorylase small subunit; GBSS『: Granule-binding starch synthase邓; SSS I: Soluble starch synthase I; PUL: Pullulanase; GPT: Glucose-6-phosphate translocator; PGIc: Plastidic glucose transporter 4; ANT: adenine nucleotide transporter; AGS: Alpha-glucosidase; GS-GOGAT: Glutamine synthetase-glutamate synthase; ARG: Argininase; ASS: Argininosuccinate; OTC: ornithine transcarbamoylase; GOT: Aspartate amino transferase; GPT: Alanine amino transferase

\section{Declarations}

Acknowledgements

The authors thank Dr. Zhixiang Luo for providing OM052 seeds.

Authors' contributions

$M X$ and WW conceived and designed the experiments; $Y X, X S, G C$ and $Y J$ performed part of the experiments; $M X$ and WW prepared the manuscript. All authors have read and approved the final manuscript.

Founding

This work was supported in part by grants from the National Natural Science Foundation (31601265), Natural Science Foundation of Anhui Province of China (1708085QC76), and the National Key Research and Development Program of China (2016YFD0300503).

Availability of data and materials

The data sets supporting the conclusions of this article are included within the article and its additional files. The data of matched proteins in Additional file 3 are from UniProt database.

(http://www.uniprot.org/uniprot/).

Ethics approval and consent to participate

Not applicable.

Consent for publication

Not applicable.

Competing interests

The authors declare that they have no competing interests. 


\section{References}

1. Xi M, Lin ZM, Zhang XC, Liu ZH, Li GH, Wang QS, et al. Endosperm structure of white-belly and whitecore rice grains shown by scanning electron microscopy. Plant Prod Sci. 2014; 17:285-90.

2. Singh N, Sodhi NS, Kaur M, Saxena SK. Physico-chemical, morphological, thermal, cooking and textural properties of chalky and translucent rice kernels. Food Chem. 2003; 82:433-39.

3. Liu XL, Guo T, Wan XY, Wang HY, Zhu MZ, Li AL, et al. Transcriptome analysis of grain-filling caryopses reveals involvement of multiple regulatory pathways in chalky grain formation in rice. BMC Genomics. $2010 ; 11: 730-44$.

4. Xi M, Zhao YL, Lin ZM, Zhang XC, Ding CQ, Tang S, et al. Comparison of physicochemical characteristics between white-belly and white-core rice grains. J Cereal Sci. 2016; 69:392-97.

5. Fitzgerald MA, Mccouch SR, Hall RD. Not just a grain of rice: the quest for quality. Trends Plant Sci. 2009; 14:133-39.

6. Lanning SB, Siebenmorgen TJ, Counce PA, Ambardekara AA, Mauromoustakoset A. Extreme nighttime air temperatures in 2010 impact rice chalkiness and milling quality. Field Crops Res. 2011; 124: 132-36.

7. Tang S, Zhang HX, Liu WZ, Dou Z, Zhou QY, Chen WZ, et al. Nitrogen fertilizer at heading stage effectively compensates for the deterioration of rice quality by affecting the starch-related properties under elevated temperatures. Food Chem. 2019; 277: 455-62.

8. Zhang H, Lei G, Zhou H, He C, Liao J, Huang Y. Quantitative iTRAQ-based proteomic analysis of rice grains to assess high night temperature stress. Proteomics. 2017; 17(5): 1-12.

9. Yamakawa $\mathrm{H}$, Hakata M. Atlas of rice grain filling-related metabolism under high temperature: joint analysis of metabolome and transcriptome demonstrated inhibition of starch accumulation and induction of amino acid accumulation. Plant Cell Physiol. 2010; 51(5): 795-809.

10. Lo PC, Hu L, Kitano H, Matsuoka M. Starch metabolism and grain chalkiness under high temperature stress. Natl Sci Rev. 2016; 3: 280-82.

11. Xiong F, Wang Z, Gu YJ, Chen G, Zhou P. Effects of nitrogen application time on caryopsis development and grain quality of rice variety Yangdao 6. Rice Sci. 2008; 15(1): 57-62.

12. Qiao JF, Liu ZH, Deng SY, Ning HF, Yang XY, Lin ZM, et al. Occurrence of perfect and imperfect grains of six japonica rice cultivars as affected by nitrogen fertilization. Plant Soil. 2011; 349: 191-202.

13. Zhou LJ, Liang SS, Ponce K, Marundon S, Ye GY, Zhao XQ. Factors affecting head rice yield and chalkiness in Indica rice. Field Crops Res. 2015; 172: 1-10. 
14. Liu LJ, Wang ZQ, Sang DZ, Yang JC. Effect of nitrogen management on rice yield and grain quality. J Yangzhou Univ. 2002; 23(3): 46-50.

15. Gu JF, Chen J, Chen L, Wang ZQ, Zhang H, Yang JC. Grain quality changes and responses to nitrogen fertilizer of japonica rice cultivars released in the Yangtze River Basin from the 1950s to 2000s. Crop J. $2015 ; 3,285-97$.

16. Kobayashi A, Genliang B, Shenghai Y, Tomita K. Detection of quantitative trait loci for white-back and basal-white kernels under high temperature stress in japonica rice varieties. Breed Sci. 2007; 57, 107-16.

17. Zhou L, Chen L, Jiang L, Zhang W, Liu L, Liu X, et al. Fine mapping of the grain chalkiness QTL qPGWC-7 in rice (Oryza sativa L.). Theor Appl Genet. 2009; 118: 581-90.

18. Mei DY, Zhu YJ, Yu YH, Fan YY, Huang DR, Zhuang JY. Quantitative trait loci for grain chalkiness and endosperm transparency detected in three recombinant inbred line populations of indica rice. $\mathrm{J}$ Integr Agr. 2013; $12(1): 1-11$.

19. Zhao DS, Li QF, Zhang CQ, Zhang C, Yang QQ, Pan LX, et al. GS9 acts as a transcriptional activator to regulate rice grain shape and appearance quality. Nat Commun. 2018; 9(1): 1240.

20. You CC, Chen L, He HB, Wu LQ, Wang SH, Ding YF, et al. iTRAQ-based proteome profile analysis of superior and inferior spikelets at early grain filling stage in japonica Rice. BMC Plant Biol. 2017; 17(1):100.

21. Juliano BO. A simplified assay for milled-rice amylose. Cereal Sci Today. 1971; 16: 334-40.

22. Isaacson T, Damasceno CM, Saravanan RS, He Y, Catalá C, Saladié M, et al. Sample extraction techniques for enhanced proteomic analysis of plant tissues. Nat Protoc. 2006; 1(2):769-74.

23. Noor E, Eden E, Milo R, Alon U. Central carbon metabolisms as a minimal biochemical walk between precursors for biomass and energy. Mol Cell. 2010; 39(5):809-20.

24. Fernie AR, Carrari F, Sweetlove LJ. Respiratory metabolism: glycolysis, the TCA cycle and mitochondrial electron transport. Curr Opin Plant Biol. 2004; 7:254-61.

25. Xu SB, Li T, Deng ZY, Chong K, Xue Y, Wang T. Dynamic proteomic analysis reveals a switch between central carbon metabolism and alcoholic fermentation in rice filling grains. Plant Physiol. 2008; 48: 90825.

26. Jeon JS, Ryoo N, Hahn TR, Walia H, Nakamura Y. Starch biosynthesis in cereal endosperm. Plant Physiol Bioch. 2010; 48, 383-92.

27. Thitisaksaku M, Jiménez RC, Arias MC, Beckles DM. Effects of environmental factors on cereal starch biosynthesis and composition. J Cereal Sci. 2012; 56: 67-80. 
28. Woo MO, Ham TH, Ji HS, Choi MS, Jiang WZ, Chu SH, et al. Inactivation of the UGPase1 gene causes genic male sterility and endosperm chalkiness in rice (Oryza sativa L.). Plant J. 2008; 54: 190-204.

29. Zakaria S, Matsuda T, Tajima S, Nitta Y. Effect of high temperature at ripening stage on the reserve accumulation in seed in some rice cultivars. Plant Prod Sci. 2002; 5:160-68.

30. Hakata M, Kuroda M, Miyashita T, Yamaguchi T, Kojima M, Sakakibara H, et al. Suppression of aamylase genes improves quality of rice grain ripened under high temperature. Plant Biotechnol J. 2012; 10:1110-17.

31. Ishimaru T, Horigane AK, Ida M, Iwasawa N, Sanoh YA, Nakazono M, et al. Formation of grain chalkiness and changes in water distribution in developing rice caryopses grown under high-temperature stress. J Cereal Sci. 2009; 50:166-74.

32. Newby AC. The role of adenosine kinase in regulating adenosine concentration. Biochem J. 1985; $226,343-44$.

33. Tjaden J, Mohlmann T, Kampfenkel K, Neuhaus GH. Altered plastidic ATP/AD- transporter activity influences potato (Solanum tuberosum L.) tuber morphology, yield and composition of tuber starch. Plant J. 1998; 16: 531.

34. Loef I, Stitt M, Geigenberger P. Increased levels of adenine nucleotides modify the interaction between starch synthesis and respiration when adenine is supplied to discs from growing potato tubers. Planta. 2001; 212: 782-91.

35. Wakasa Y, Yasuda H, Oono Y, Kawakatsu T, Hirose S, Takahashi H, et al. Expression of ER quality control-related genes in response to changes in BiP1 levels in developing rice endosperm. Plant J. 2011; 65:675-89.

36. Wahl MC, Moller W. Structure and function of the acidic ribosomal stalk proteins. Curr Protein Pept Sci. 2002; 3: 93-106.

37. Mayer MP, Bukau B. "Hsp70 chaperones: Cellular functions and molecular mechanism". Cell. Mol. Life Sci. 2005; 62 (6): 670-84.

38. Satoh-Cruz M, Crofts AJ, Takemoto-Kuno Y, Sugino A, Washida H, Crofts N, et al. Protein disulfide isomerase like 1-1 participates in the maturation of proglutelin within the endoplasmic reticulum in rice endosperm. Plant Cell Physiol. 2010; 51(9): 1581-93.

39. Han XH, Wang YH, Liu X, Jiang L, Ren YL, Liu F, et al. The failure to express a protein disulphide isomerase-like protein results in a floury endosperm and an endoplasmic reticulum stress response in rice. J Exp Bot. 2012; 63(1): 121-30. 
40. Kim YJ, Yeu SY, Park BS, Koh HJ, Song JT, Seo HS. Protein disulfide isomerase-like protein 1-1 controls endosperm development through regulation of the amount and composition of seed proteins in rice. Plos One. 2012; 7(9): e44493.

41. Lin SK, Chang MC, Tsai YG, Lur HS. Proteomic analysis of the expression of proteins related to rice quality during caryopsis development and the effect of high temperature on expression. Proteomics. 2005; 5(8): 2140-56.

42. Hellmann H. Plant development: regulation by protein degradation. Science. 2002; 297(5582):79397.

43. Kurepa J, Smalle JA. Structure, function and regulation of plant proteasomes. Biochimie, 2008; 90(2): 324-35.

44. Lin Z, Zhang X, Yang X, Li GH, Tang S. Wang SH, et al. Proteomic analysis of proteins related to rice grain chalkiness using iTRAQ and a novel comparison system based on a notched-belly mutant with white-belly. BMC Plant Biol. 2014; 14(1): 163.

45. Washida H, Sugino A, Doroshenk KA, Satoh-Cruz M, Nagamine A, Katsube-Tanaka T, et al. RNA targeting to a specific ER sub-domain is required for efficient transport and packaging of a-globulins to the protein storage vacuole in developing rice endosperm. Plant J. 2012; 70, 471-79.

46. Ren Y, Wang Y, Liu F, Zhou K, Ding Y, Zhou F, et al. GLUTELIN PRECURSOR ACCUMULATION3 Encodes a regulator of post-golgi vesicular traffic essential for vacuolar protein sorting in rice endosperm. Plant Cell. 2014; 26, 410-25.

47. Lin CJ, Li CY, Lin SK, Yang FH, Huang JJ, Liu YH, et al. Influence of high temperature during grain filling on the accumulation of storage proteins and grain quality in rice (Oryza sativa L.). J Agric Food Chem. 2010; 58 (19): 10545-52.

48. Kawakatsu T, Hirose S, Yasuda H, Takaiwa F. Reducing rice seed storage protein accumulation leads to changes in nutrient quality and storage organelle formation. Plant Physiol. 2010; 154: 1842-54.

49. Hattori A. Studies on the metabolism of urea \& other nitrogenous compounds in Chlorella ellipsoidea. I. Assimilation of urea \& other nitrogenous compounds by nitrogeni-starved cells. J. Biochen. (Tokyo) 1957; 44: 253-73.

50. Winter G, Todd CD, Trovato M, Forlani G, Funck D. Physiological implications of arginine metabolism in plants. Front Plant Sci. 2015; 6: 534.

51. Unno H, Uchida T, Sugawara H, Kurisu G, Sugiyama T, Yamaya T, et al. Atomic structure of plant glutamine synthetase: a key enzyme for plant productivity. J Biol Chem. 2006; 281: 29287-96. 
52. Xu GH, Fan XR, Miller AJ. Plant nitrogen assimilation and use efficiency. Annu Rev Plant Biol. 2012; 63:153-82.

53. Zhao XQ, Shi WM. Expression analysis of the glutamine synthetase and glutamate synthase gene families in young rice (Oryza sativa) seedlings. Plant Sci. 2006; 170:748-54.

54. Duan MJ, Sun SSM. Profiling the expression of genes controlling rice grain quality. Plant Mol Biol. 2005; 59: 165-78.

\section{Tables And Additional Files}

Due to technical limitations, the tables are only available as a download in the supplemental files section.

Additional file 1: Figure S1. Image of dehulled grains of OM052

Additional file 2: Figure S2. Daily average air temperature and precipitation over rice growing seasons in 2016 and 2017.

Additional file 3: Table S1. List of all proteins identified and quantified by nano-HPLC/MS/MS

Additional file 4: Table S2. Primers for qRT-PCR assay.

\section{Figures}
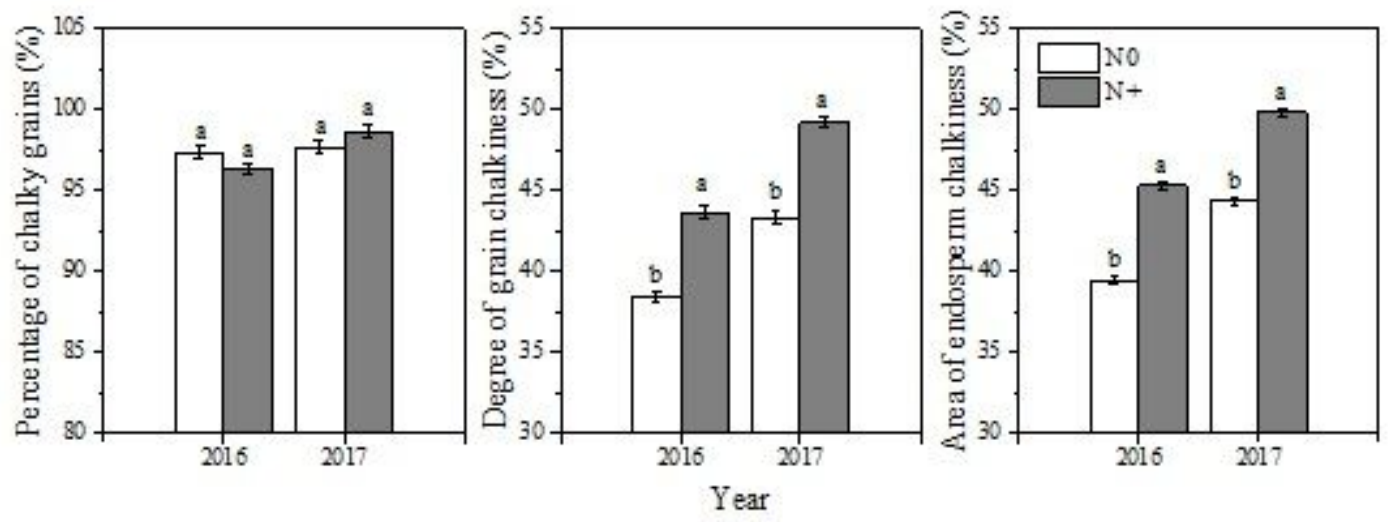

Fig.1 Effects of $\mathrm{N}$ topdressing on rice appearance quality. $\mathrm{PCG}$, percentage of chalky grains; DGC, degree of grain chalkiness; $\mathrm{AEC}$, area of endosperm chalkiness

\section{Figure 1}

Effects of $\mathrm{N}$ topdressing on rice appearance quality. PCG, percentage of chalky grains; DGC, degree of grain chalkiness; AEC, area of endosperm chalkiness 


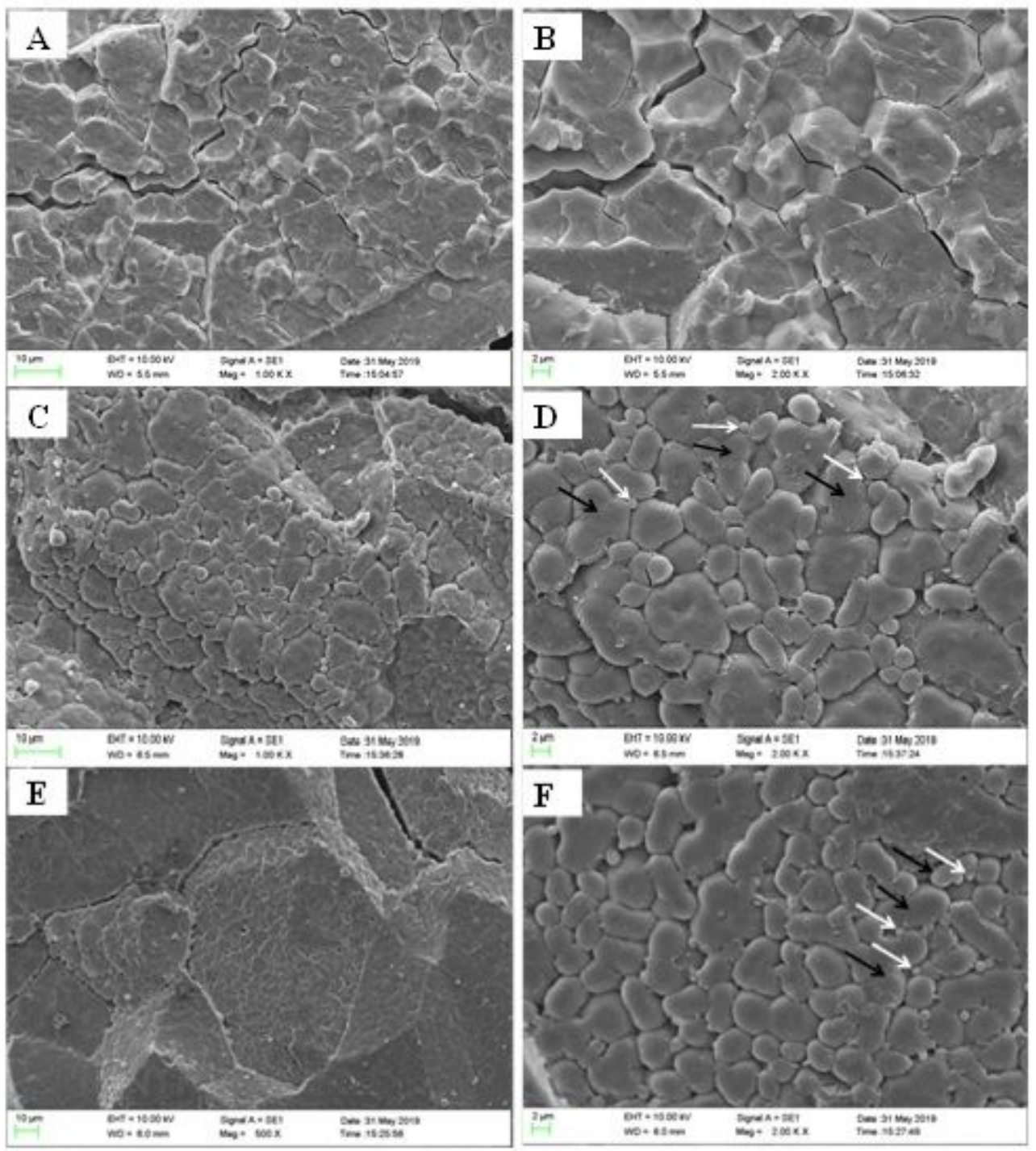

Fig.2 SEM images of grain endosperm structure. a and (b) Endosperm structure of translucent grains; $\mathbf{c}$ and (d) Endosperm structure of grains from the control of $\mathrm{N} 0$ treatment; $e$ and (f)Endosperm structure of grains exposed to $\mathrm{N}+$ treatment. Black arrows indicate the starch granules, and white arrows indicate protein bodies accumulated around the starch granules. Note that there are air spaces between starch granules and protein bodies

\section{Figure 2}

SEM images of grain endosperm structure. a and (b) Endosperm structure of translucent grains; $c$ and (d) Endosperm structure of grains from the control of NO treatment; e and (f)Endosperm structure of grains exposed to $\mathrm{N}+$ treatment. Black arrows indicate the starch granules, and white arrows indicate protein bodies accumulated around the starch granules. Note that there are air spaces between starch granules and protein bodies 


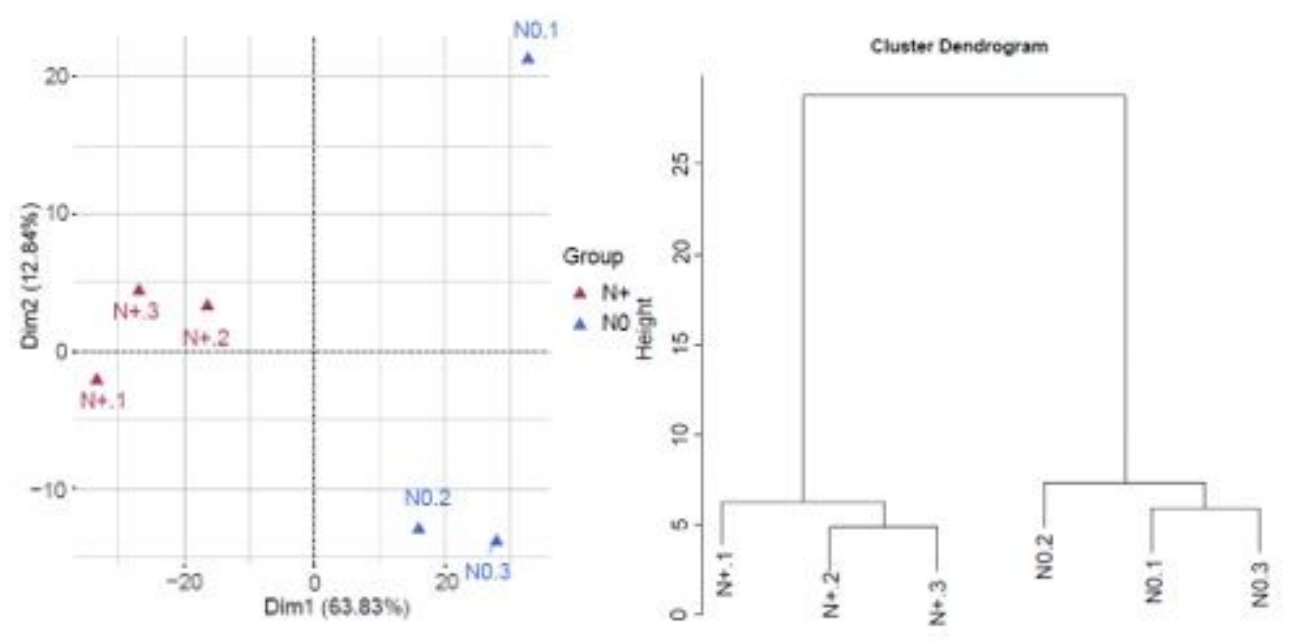

Fig.3 Principal component (PCA) and cluster analysis of differential expressed proteins using individual replicates. a Principal component analysis (PCA). Data are expressed as relative values: $\mathrm{N}+$ is shown in green, and $\mathrm{N} 0$ is shown in blue. $\mathbf{b}$ The individual replicates were separated into two groups

\section{Figure 3}

Principal component (PCA) and cluster analysis of differential expressed proteins using individual replicates. a Principal component analysis (PCA). Data are expressed as relative values: $\mathrm{N}+$ is shown in green, and NO is shown in blue. b The individual replicates were separated into two groups 


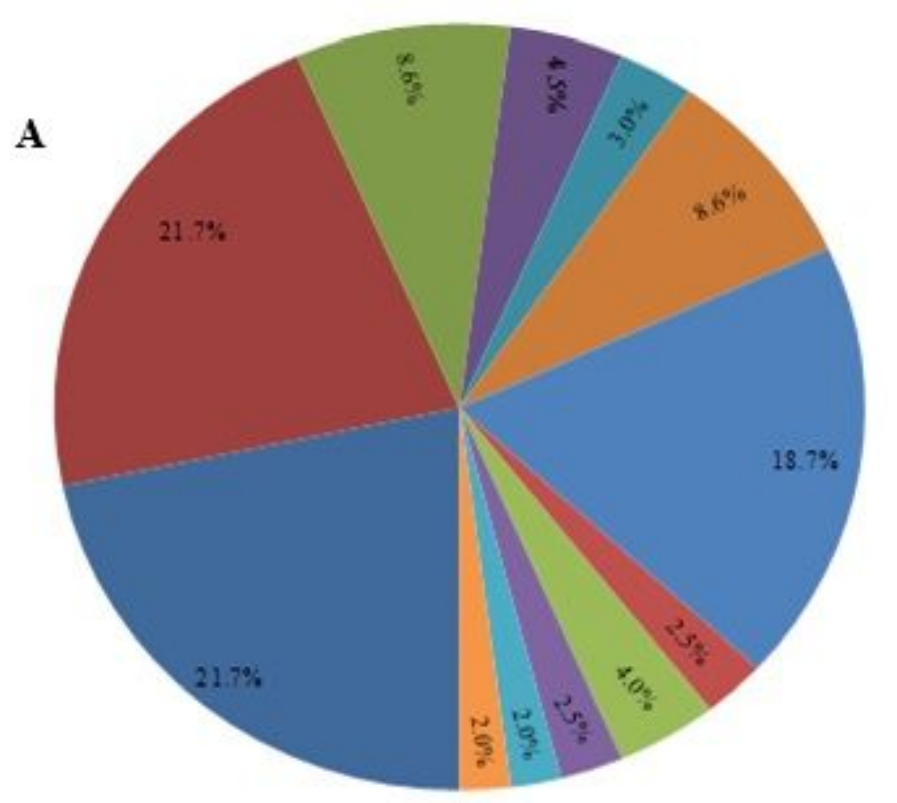

n Prote in metabolism

- Casbohydrate metabolism

e Am mo acid m etabolism

= Photo synthe sis and electron tran rfer

n Lipid metabolism

nell growth/division

n Stress and defen se

n Energy m etabolism

Material transport

= Signal transduction

- Phosphorus as etabolism

Unknown

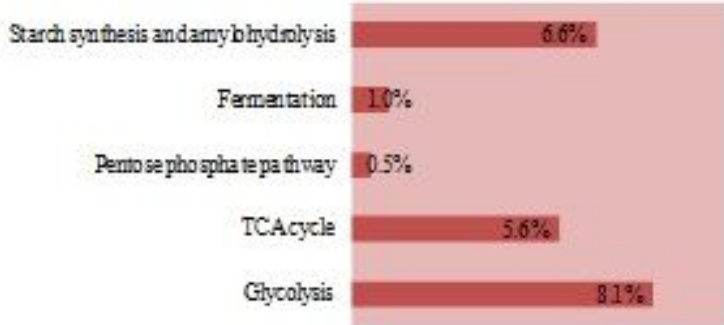

B

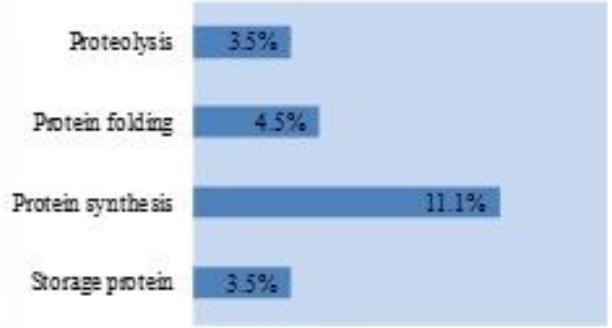

C

Fig. 4 Functional classifications of the differentially expressed proteins. a Total differentially expressed proteins (198). b Proteins involved in carbohydrate metabolism(43). c Proteins involved innitrogen metabolism(45)

\section{Figure 4}

Functional classifications of the differentially expressed proteins. a Total differentially expressed proteins (198). b Proteins involved in carbohydrate metabolism(43). c Proteins involved innitrogen metabolism(45) 


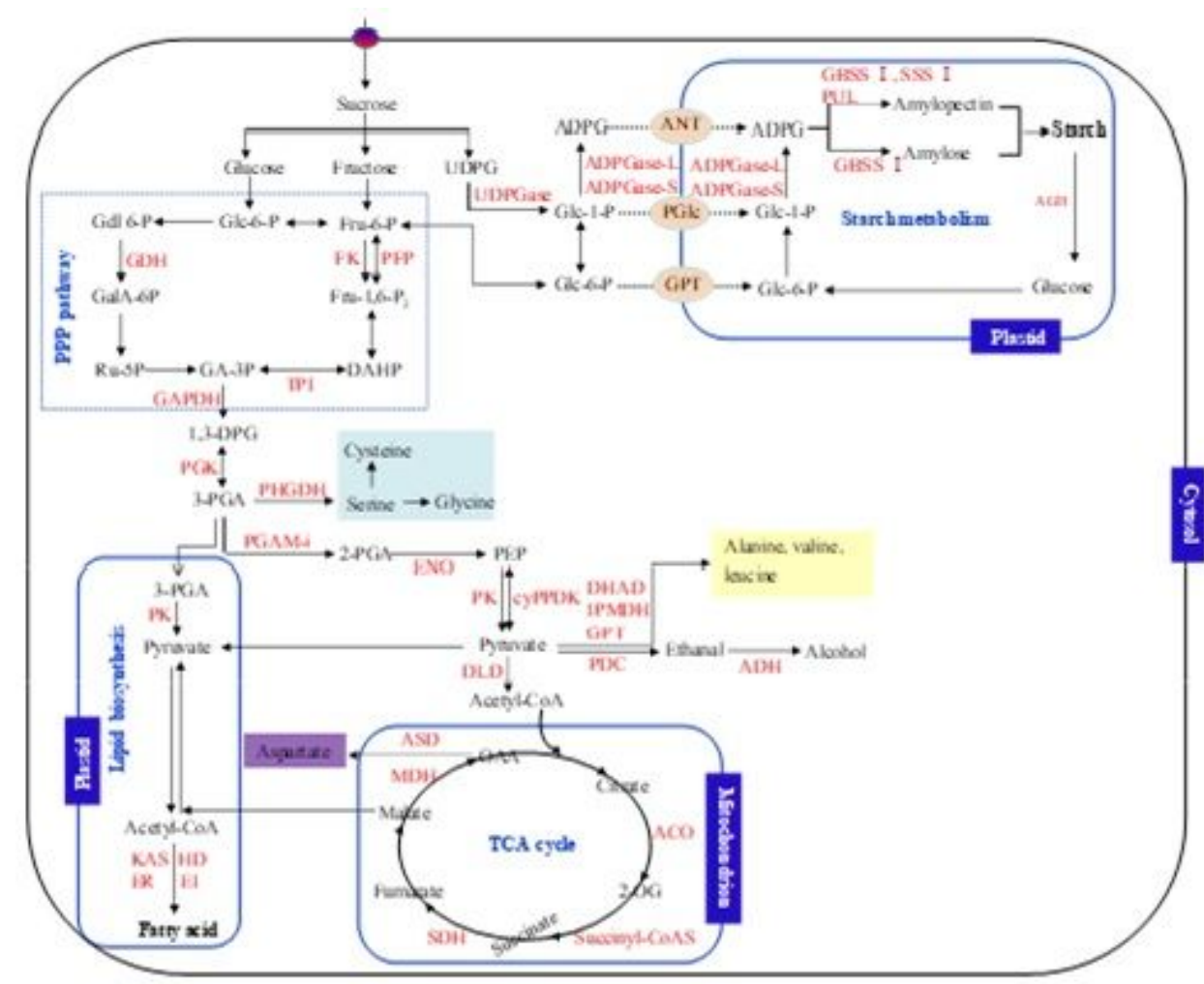

Fig. 5 Overview of differentially expressed proteins on carbohydrate metabolism. Up-regulated proteins are designated in red font. Glc, glucose; Fru, fructose; UDPG, uridinediphosphate glucose; P, phosphate; Gdl, gluconsolactone; GalA, galacturonic acid; Ru-5P, ribulose 5-phosphate; GA-3P, glyceradehycle 3-phosphate; DHAP, dihydroxyacetone phosphate; 1,3-DPG, 1, 3-diphosphoglycerate; 3-PGA, 3-phosphoglycerate; 2-PGA, 2-phosphoglycerate; PEP, phosphoenolpyruvate; GDH, glucose dehydrogenase; FK, fructokinase; ENO, enolase; PFP, pyrophosphate-fructose 6-phosphate 1-phosphotransferase; TPI, triosephosphateisomerase; GAPDH, glyceraldehyde-3-phosphate dehydrogenase; PGK, phosphoglycerate kinase; PHGDH,D-3-phosphoglycerate dehydrogenase; PGAM-i, 2,3-bisphosphoglycerate independent phosphoglyceratemutase; PK, Pyruvate kinase; DHAD, dihydroxylatedehydrase; IPMDH, 3-isopropylmalate dehydrogenase; GPT, alanine aminotransferase 2; PDC, pyruvate decarboxylase 2; ADH, alcohol dehydrogenase; DLD, dihydrolipoamide dehydrogenase; ACO, aconitase; Succinyl-CoAS, succinyl-CoA synthase; SDH, succinate dehydrogenase; $\mathbf{M D H}$, malate dehydrogenase; ASD, aspartate-semialdehyde dehydrogenase; KAS, 3-oxoacyl-[acyl-carrier-protein] synthase; HD, beta-hydroxyacyl-ACP dehydratase; ER , enoyl-[acyl-carrier-] reductase; EI, enoyl-CoA delta isomerase; OAA, oxaloacetate; 2-OG, 2-oxoglutarate; ACP, acyl carrier protein; UDPGase,UDP-glucose pyrophosphorylase; ADPG, adenosine diphosphate glucose; ADPGase-L, ADP-glucose pyrophosphorylase large subunit; ADPGase-S, ADP-glucose pyrophosphorylase small subunit; GBSSI, granule-binding starch synthase I ; SSS I, soluble starch synthase I ;PUL, pullulanase; AGH, alpha-glucosidase; GPT, glucose-6-phosphate translocator; ANT, adenylate transporter; PGlc, Plastidic glucose transporter 4; TCA cycle, tricarboxylic acid cycle; cyPPDK, cytosolic pyruvate orthophosphate dikinase; PPP, pentose phosphate pathway

\section{Figure 5}

Overview of differentially expressed proteins on carbohydrate metabolism. Up-regulated proteins are designated in red font. Glc, glucose; Fru, fructose; UDPG, uridinediphosphate glucose; P, phosphate; Gdl, gluconsolactone; GalA, galacturonic acid; Ru-5P, ribulose 5-phosphate; GA-3P, glyceradehycle 3phosphate; DHAP, dihydroxyacetone phosphate; 1,3-DPG, 1, 3-diphosphoglycerate; 3-PGA, 3phosphoglycerate; 2-PGA, 2-phosphoglycerate; PEP, phosphoenolpyruvate; GDH, glucose dehydrogenase; 
FK, fructokinase; ENO, enolase; PFP, pyrophosphate-fructose 6-phosphate 1-phosphotransferase; TPI, triosephosphateisomerase; GAPDH, glyceraldehyde-3-phosphate dehydrogenase; PGK, phosphoglycerate kinase; PHGDH,D-3-phosphoglycerate dehydrogenase; PGAM-i, 2,3-bisphosphoglycerate independent phosphoglyceratemutase; PK, Pyruvate kinase; DHAD, dihydroxylatedehydrase; IPMDH, 3-isopropylmalate dehydrogenase; GPT, alanine aminotransferase 2; PDC, pyruvate decarboxylase 2; $A D H$, alcohol dehydrogenase; DLD, dihydrolipoamide dehydrogenase; ACO, aconitase; Succinyl-CoAS, succinyl-CoA synthase; SDH, succinate dehydrogenase; $\mathrm{MDH}$, malate dehydrogenase; ASD, aspartate-semialdehyde dehydrogenase; KAS, 3-oxoacyl-[acyl-carrier-protein] synthase; HD, beta-hydroxyacyl-ACP dehydratase; ER ,enoyl-[acyl-carrier-] reductase; El, enoyl-CoA delta isomerase; OAA, oxaloacetate; 2-OG, 2-oxoglutarate; ACP, acyl carrier protein; UDPGase,UDP-glucose pyrophosphorylase; ADPG, adenosine diphosphate glucose; ADPGase-L, ADP-glucose pyrophosphorylase large subunit; ADPGase-S, ADP-glucose

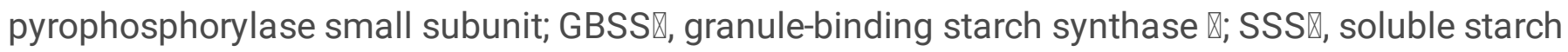
synthase邓;PUL, pullulanase; AGH, alpha-glucosidase; GPT, glucose-6-phosphate translocator; ANT, adenylate transporter; PGIc, Plastidic glucose transporter 4; TCA cycle, tricarboxylic acid cycle; cyPPDK, cytosolic pyruvate orthophosphate dikinase; PPP, pentose phosphate pathway

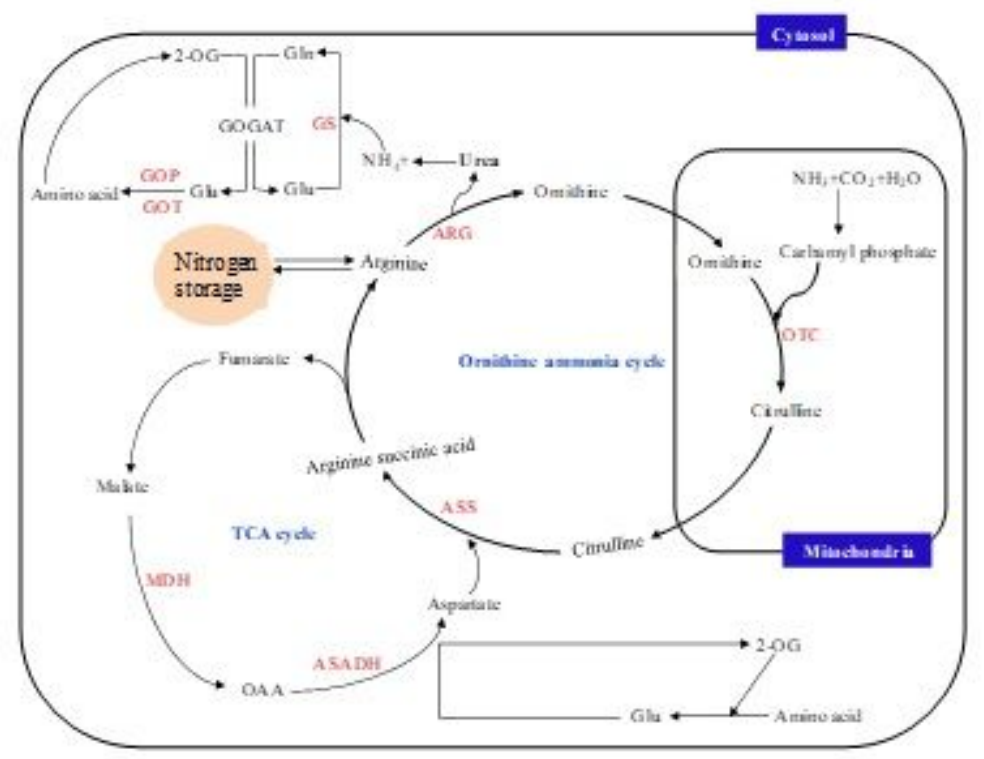

Fig.6 Differentially expressed proteins associated with omithine cycle. Glu, Glutamate; Gln, Glutamine; 2-OG, 2-oxoglutarate; OAA, oxaloacetate; $\mathbf{M D H}$, malate dehydrogenase; GPT, alanine aminotransferase 2; GOT, Aspartate aminotransferase; ARG, argininase; OTC,omithine carbamoyltransferase; ASS, argininosuccinatechloroplastic; GS, glutamine synthetase; ASADH, aspartate-semialdehyde dehydrogenase; GOGAT, Glutamate synthase

\section{Figure 6}

Differentially expressed proteins associated with ornithine cycle. Glu, Glutamate; Gln, Glutamine; 2-OG, 2oxoglutarate; OAA, oxaloacetate; MDH, malate dehydrogenase; GPT, alanine aminotransferase 2; GOT, Aspartate aminotransferase; ARG,argininase; OTC,ornithine carbamoyltransferase; ASS, 
argininosuccinatechloroplastic; GS, glutamine synthetase; ASADH, aspartate-semialdehyde dehydrogenase; GOGAT, Glutamate synthase

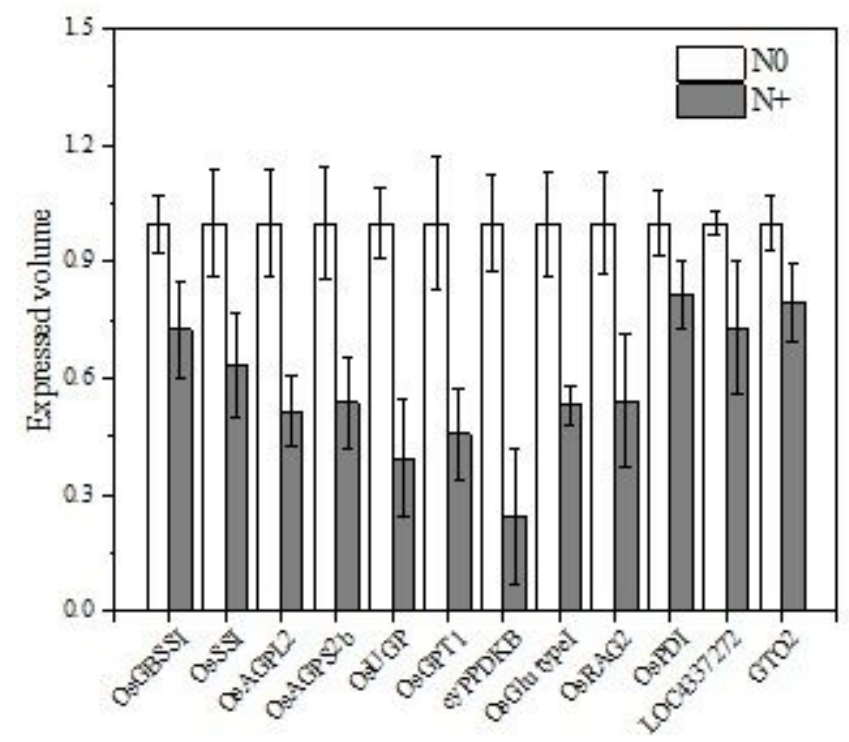

Fig.7 RT-PCR confirmation of proteome data. Twelve differentially expressed proteins were randomly selected for RT-PCR analysis

Figure 7

RT-PCR confirmation of proteome data. Twelve differentially expressed proteins were randomly selected for RT-PCR analysis

\section{Supplementary Files}

This is a list of supplementary files associated with this preprint. Click to download.

- Additionalfile3TableS1.xlsx

- Tables.pdf

- Additionalfile1Figures1.pdf

- Additionalfile2Figures2.pdf

- Additionalfile4Tables2.xIsx 